herniation of the brainstem through the foramen magnum. Sarnat proposes a molecular genetic theory of pathogenesis due to mutation of candidate genes such as HOX, WNT, and PAX, with ectopic expression in the embryonic hindbrain. Vimentin immunoreactivity in the ependyma may provide a useful marker of focal abnormality in the fourth ventricle and aqueduct in fetuses and infants with congenital hydrocephalus.

Chiari I malformation and neurofibromatosis type 1. Two large groups of pediatric patients at the Children's Hospital, University of Alabama, Birmingham, AL were examined retrospectively to determine the significance of an association of these conditions. Of 130 surgically treated Chiari I malformations (Group 1), $5.4 \%$ also had neurofibromatosis type 1. Of a second group of 198 patients seen in the neurofibromatosis clinic and who underwent neuroimaging, $8.6 \%$ had a concomitant Chiari I malformation. The authors hypothesize that the same early dysgenesis of mesoderm responsible for Chiari I malformation may also account for neurofibromatosis type 1. (Tubbs RS, Rutledge SL, Kosentka A, et al. Pediatr Neurol 2004;30:278-280).

For further accounts of recent advances in cerebral dysgenesis, see Sarnat HB, in Progress in Pediatric Neurology III, PNB Publishers, 1997;365-369; Sarnat HB. Gene table. CNS malformations: gene locations of known human mutations. Eur J Pediatr Neurol 2004;8:105-108; and Sarnat HB. Watershed infarcts in the fetal and neonatal brainstem. An etiology of central hypoventilation, dysphagia, Mobius syndrome and micrognathia. Eur J Pediatr Neurol 2004;8:71-87.) Also, see Sheen VL, Basel-Vanagaite L, Goodman JR, et al. Etiological heterogeneity of familial periventricular heterotopia and hydrocephalus. Brain Dev 2004;26:326-334. One pedigree had a known FLNA mutation with hydrocephalus and valproate exposure during pregnancy. Most neural tube defects arise from multifactorial causes, with interactions between genetic and environmental factors.

\title{
FAMILIAL CONGENITAL FACIAL PALSY
}

Three males with congenital facial palsy from 3 generations in the same family are reported from the Floating Hospital for Children, Tufts-New England Medical Center, Boston, MA. Each had a left facial palsy, more severe in successive generations. The proband, a 9-year-old male, and his father had abnormal MRI studies, with enlargement and/or enhancement of the tympanic portion of the facial nerve. Taste in the anterior two thirds of the tongue was preserved, and blink reflexes were asymmetric. EMG/nerve conduction studies confirmed the nerve palsy and showed lowered compound muscle action potential amplitudes. The etiology was undetermined. (Kondev L, Bhadelia RA, Douglass LM. Familial congenital facial palsy. Pediatr Neurol 2004;30:367-370). (Respond: Dr Kondev, Floating Hospital for Children at Tufts-New England Medical Center, Division of Pediatric Neurology, 750 Washington St, Tufts-NEMC \#330, Boston, MA 02111).

COMMENT. This appears to be the first report of familial congenital facial palsy. A partial agenesis of the facial nerve nucleus is considered in etiology. In the absence of an associated sixth nerve palsy, a diagnosis of Mobius syndrome is unlikely. 